\title{
Advancements in Raman technology for identifying real-world samples
}

\section{Jun Zhao, Katherine Bakeev, Xin Jack Zhou}

Jun Zhao, Katherine A. Bakeev, Xin Jack Zhou, "Advancements in Raman technology for identifying real-world samples," Proc. SPIE 10983, NextGeneration Spectroscopic Technologies XII, 109830L (13 May 2019); doi: $10.1117 / 12.2521151$

Event: SPIE Defense + Commercial Sensing, 2019, Baltimore, MD, United States 


\title{
Advancements in Raman technology for identifying real-world samples \\ Jun Zhao*, Katherine A. Bakeev, Xin Jack Zhou \\ B\&W Tek LLC, 19 Shea Way Suite 301, Newark, DE 19713, USA
}

\begin{abstract}
Handheld Raman spectroscopy's value for rapid field screening by safety and security personnel is well understood as evidenced by its implementation across the globe. Though Raman spectroscopy has the ability to nondestructively identify samples through transparent packaging, in real world scenarios challenging samples are frequently encountered. Raman screening must be effective for samples in a variety of packaging and for samples with coloration or impurities that give a high fluoresce that can overwhelm the Raman signal depending on the excitation laser wavelength. See through Raman technology has been developed to enable measurement through opaque packaging, sampling a larger area and with a deeper penetration depth of the Raman signal. Using a design of collinear sample illumination and Raman scattering collection at a higher efficiency, and spread over a larger sampling area, there is a lower power density of illumination on the sample, reducing issues of sample heating that can be problematic for dark samples. The measurement of a larger sample area provides more reliable identification of solids that are often inhomogeneous. This see through Raman technology and the use of a longer laser wavelength excitation overcomes many of the difficulties encountered in use of Raman spectroscopy for field testing.
\end{abstract}

Keywords: Raman spectroscopy, field testing, see through analysis, chemical identification, reduced power density, sample screening, large sample area

\section{INTRODUCTION}

Raman spectroscopy is a non-destructive spectroscopic technique for material characterization and identification of solids, liquids and semi-solids. Over the past two decades Raman instrumentation has become more compact, and the technology in a handheld instrument format is increasingly used for analysis by law enforcement, customs and border protection, and the pharmaceutical industry. ${ }^{1,2}$ Because of its ease of use, rapid results and ability to identify many materials with minimal contact of the sample by a user, the safety and security sector increasingly utilizes Raman as a field screening tool for substances wherever they may encountered. The capability for sample screening in place allows for rapid response and minimizes exposure to potential hazards. Effective Raman field screening requires an easily operable system with minimal data interpretation. A field Raman system should have the capability to identify samples in a variety of packaging, as well as dark or impure samples.

Typically Raman measures over a small sample area at the laser focus point, which has drawbacks both due to the area of measurement that limits the ability to make a representative measurement of nonhomogeneous samples, and because of the high power density of the laser spot on the small area which concentrates energy and may cause sample heating. Development of a Raman device with see through technology that overcomes many of these challenges, especially when used with a longer laser excitation wavelength opens the door for even greater utility of handheld Raman spectroscopy. ${ }^{3}$, ${ }^{4}$ The design of a Raman sampling device with improved light delivery and collection intended for measurement of a large sample area, and at a deeper depth of penetration is presented, with examples illustrating the advantages in identifying real-world samples. The design further entails an optimized spectrograph to effectively collect the greater number of photons delivered from the optical system.

Raman spectroscopy using a $1064 \mathrm{~nm}$ near-infrared laser wavelength excitation reduces the interference of fluorescence that plagues identification of dark and colored samples when using a shorter wavelength. The combined technology of 1064-nm excitation and a see through (ST) reflective cavity design gives higher signal throughput to partially overcome the decreased scattering efficiency at the longer wavelength without having to increase measurement time. The see through optical design used with the 1064-nm laser Raman system has the already mentioned benefits of larger sample area, lower power density and ability to also measure samples through packaging layers that fluoresce such as kraft paper bags.

*junz@bwtek.com; phone 1302368 7824; www.bwtek.com

Next-Generation Spectroscopic Technologies XII, edited by Richard A. Crocombe, Luisa T. M. Profeta, Abul K. Azad, Proc. of SPIE Vol. 10983, 109830L · @ 2019 SPIE · CCC code: 0277-786X/19/\$18 · doi: 10.1117/12.2521151 
A similar technology of spatially offset Raman spectroscopy (SORS) can measure a Raman spectrum through opaque layers while largely avoiding the Raman spectrum of these layers from overwhelming that of the sample. ${ }^{5}$ SORS operates by intentionally offsetting the excitation beam and collection beam while the technology described here uses a collinear sample illumination and Raman scattering collection with high efficiency signal collection. The SORS design cannot be easily used in a confocal arrangement and are generally inefficient for direct sample measurements. The commercially available SORS system uses an $830 \mathrm{~nm}$ laser excitation, which is impacted by sample fluorescence similar to a $785 \mathrm{~nm}$ Raman system.

\section{SEE THROUGH RAMAN SYSTEM DESIGN}

In conventional Raman instrumentation the sampling optics are designed with a focused design similar to confocal microscopes. They are designed with both the excitation beam and the Raman signal beam focused at the same spot. The sample is usually placed at this focal point and has a small sampling area with high power density at the laser focal point. In this way, excitation power density and consequently the Raman signal radiance are maximized at the sampled volume, and only signal from this tightly focused volume is collected. This confocal design has the advantage of maximum throughput and can be used to measure samples inside transparent containers in the same way a confocal microscope does optical sectioning. The confocal approach loses its effectiveness when the container diffuses the light strongly because the light can no longer be focused onto the materials inside the container. The Raman signal for materials inside diffusely scattering containers is weak and is often accompanied by strong features from the container itself.

The STRaman ${ }^{\circledR}$ technology expands the capability of Raman spectroscopy to measure samples beneath diffusely scattering packaging material - allowing a see through (ST) identification of samples inside opaque packages as well as transparent layers which can be done with conventional Raman. ST Raman technology is based on increased light delivery and collection system for Raman by the use of a reflective cavity. The multiple reflections of the Raman scattering from a sample increases the excitation efficiency and also enhances the collection efficiency, and is used with a spectrograph optimized to collect the extra photons delivered from the reflective cavity.

The STRaman ${ }^{\circledR}$ reflective cavity serves three purposes optically giving multiple enhancements over conventional Raman spectroscopic analysis: (1) providing a larger sampling area than the confocal approach; (2) maximizing signal collection by means of multiple reflections and scattering, and (3) isolating the sampled area from ambient light which would contaminate the signal. The use of the specialized reflective cavity enhances the relative intensity of the Raman signal from the deeper layers, thereby increasing the effective sampling depth, and allows the measurement of material inside visually opaque containers. The larger sampling area has the added advantage of reducing the power density of the laser on the sample and thus eliminating sample damage. With this decreased power density colored samples and energetic material can be measured without sample damage from the laser burning the surface. The large sampling area improves measurement accuracy by eliminating the variability detected when measuring with a small spot size on a heterogeneous sample where the spot size is on the order of the grain size.

\subsection{See through identification}

In conventional Raman, the excitation laser is focused on or close to the sample surface, as shown in Figure 1(a). When this surface is non-transparent packaging such as a white polyethylene (PE) bottle, the Raman scattering from the bottle surface is strong, and only a small amount of the laser penetrates through the wall to the sample inside. The spectrum 1(a) below of is of a sample of sodium benzoate in the white bottle, where the arrows are pointing to the weak signal of the sodium benzoate, with the predominant signal arising from the Raman scattering of the PE bottle. Using a defocused excitation beam and a diffuse scattering mechanism, the light is covering a larger area (several $\mathrm{mm}$ ) and penetrates through the bottle wall. In this case the Raman scattering of the sample beneath the surface is measured with less of a contribution from the surface itself. The spectrum in 1(b) has the signal of the sodium benzoate as the predominant peaks, though their overall intensity is not that high, and the bottle surface has a much smaller overall contribution. Using the reflective cavity of the STRaman technology as shown schematically in figure 1(c), the Raman scattering signal as well as the excitation signal are enhanced by being reflected in the cavity and having multiple bounces on the sample, over the larger sample area and to a greater depth. The STRaman technology results in a Raman spectrum with 
overall greater intensity dominated by the subsurface sodium benzoate peaks, marked with arrows in figure 1(c), with minor contribution from the PE bottle surface. The reflective cavity increases the signal throughout almost $8 \mathrm{x}$.

(a)
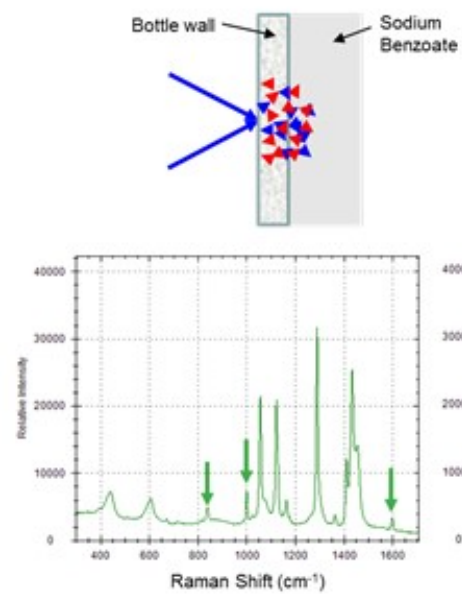

(b)
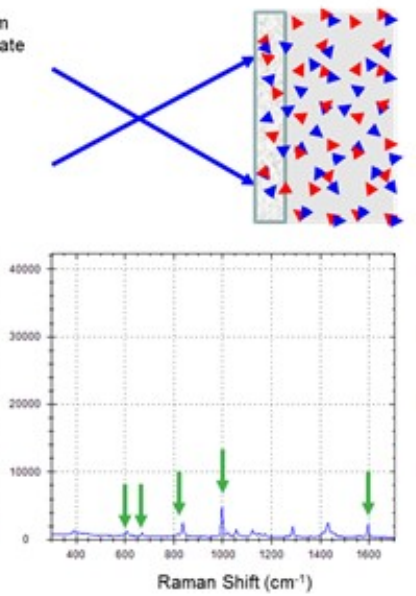

(c)
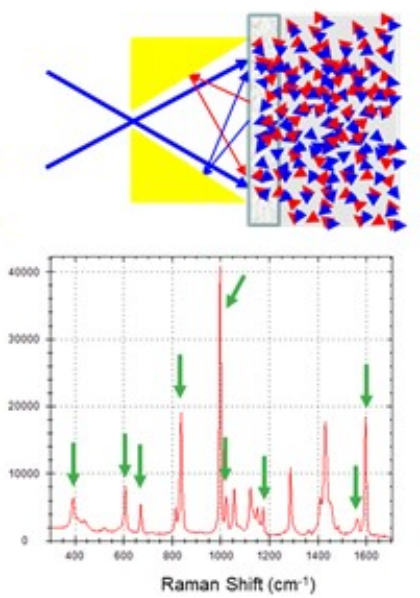

Figure 1: Spectra of sodium benzoate in a white polyethylene bottle in the (a) standard Raman configuration; (b) defocused laser excitation covering larger area, deeper volume of sample and (c) with reflective cavity of STRaman technology with much enhanced signal over the larger area and beneath surface. (785 nm laser excitation, $1 \mathrm{sec}$ integration, $\sim 300 \mathrm{~mW}$ laser power).

This example illustrates the ability for subsurface sampling such that sample can be identified with minimal handling even when the packaging is opaque. This is clearly beneficial in minimizing the need to perturb samples, and identify samples for quality control, and to detect mislabeled and unknown materials as may be encountered by first responders, customs, postal service and shipping companies.

The identification of samples such as coated tablets is important within the safety industry, and by customs and regulators for the detection of controlled substances as well as counterfeits. Tablets are often coated with sugar and with colorants or titanium dioxide to make them more palatable, increase tablet durability, control the dissolution properties, and to distinguish the product with distinctive branding. In order to identify the tablet's active ingredient (s) it is necessary to break the tablet, remove the coating layer or have a method to measure through the tablet coating. The spectrum in Figure 2c of an Advil ${ }^{\circledR}$ tablet with the coating removed shows the clear distinct Raman spectrum of ibuprofen. Using library search algorithms gives users a rapid means of identifying the sample as ibuprofen. When this enteric-coated tablet was scanned by conventional Raman scattering, the major contribution to the spectrum is the sucrose coating, where the peaks in Figure 2(b) from 600 to $1400 \mathrm{~cm}^{-1}$ are attributable to the sucrose, while the peaks of the active are absent. The see through scan of the coted table shown in Figure 2(a) has contributions of both the active ibuprofen with small peaks of sucrose also visible, The strong signal of the ibuprofen, marked by arrows has a close resemblance with the bottom spectrum where the coating is removed. Specialized library searching methods using proprietary algorithms have been developed to isolate the sub-surface Raman spectrum from the surface spectrum so that the sample can be identified, without confounding results from the packaging or other surface layer signal.

An additional benefit of this STRaman system is that even colored tablet coatings can be measured without sample surface burning due to the lower power density of the system relative to that of the highly focused signal used in conventional Raman. 


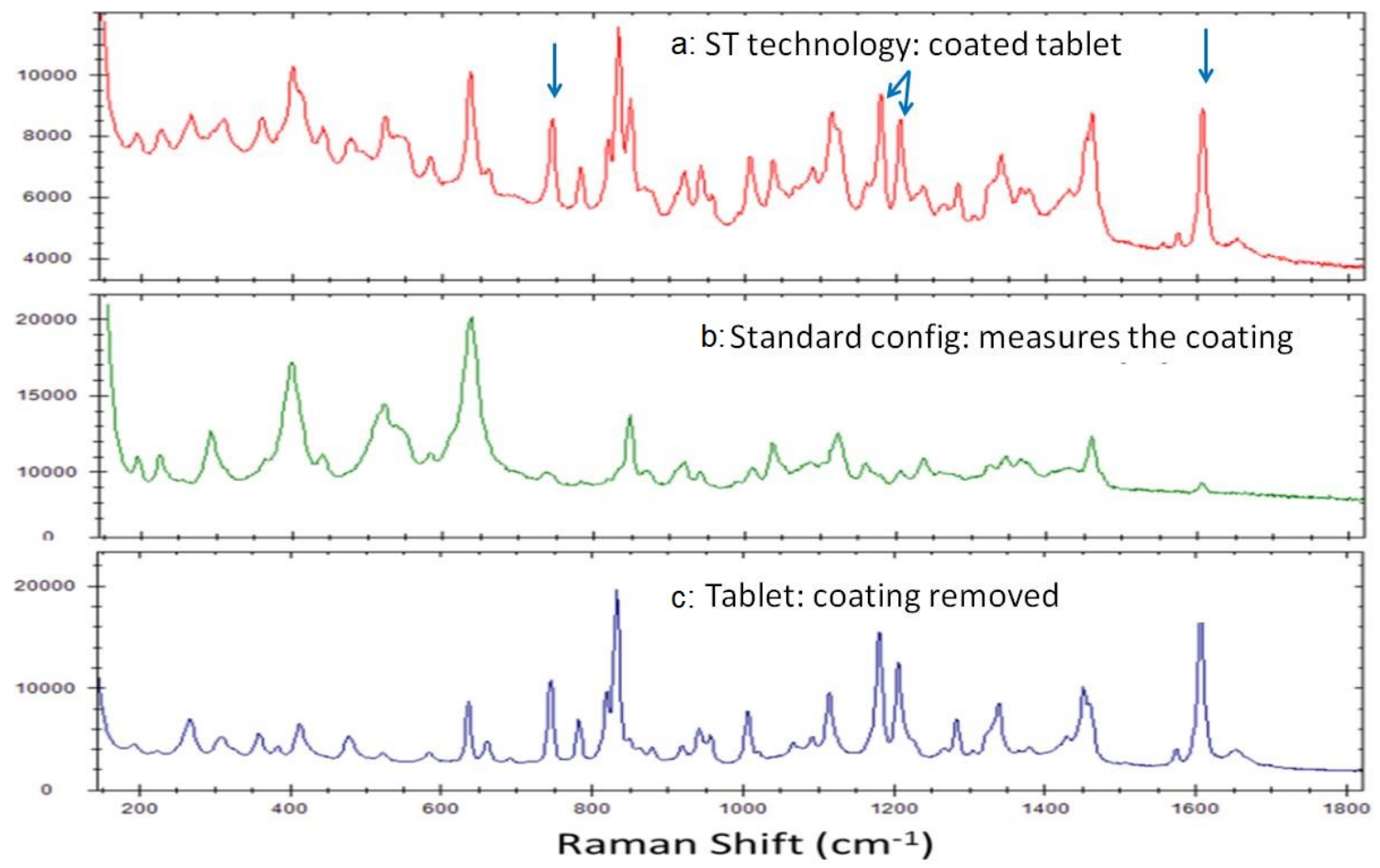

Figure 2: Raman spectra of coated tablets. (a) Coated tablet spectrum measured using the STRaman technology; (b) Coated tablet spectrum measured with a conventional confocal Raman configuration; (c) spectrum of the tablet with the coating stripped measured with the conventional confocal configuration.

\subsection{4-nm laser STRaman for sampling through fluorescing layers}

There are many cases in which multiple layers of packaging are used for samples, including street trade of illicit substances. Many raw materials for pharmaceutical and consumer products are supplied in kraft paper sacks of single or multiple layers, sometimes with a plastic lining layer as well. Brown kraft paper, made from chemical pulp, has a strong fluorescence when measured with $785 \mathrm{~nm}$ Raman as do many other dark colored materials. The increased penetration achieved with the STRaman technology on a $1064 \mathrm{~nm}$ Raman system enables material identification through such challenging surface layers. This technology has been used to identify materials from weak Raman scatterers such as trisodium phosphate to the strongly Raman active calcium carbonate through different multi-layer paper bags. Successful identification has been made based on the Raman spectrum measured through bags with multiple layers of kraft paper, white paper and plastic film.

The $1064 \mathrm{~nm}$ Raman spectrum of trisodium phosphate $\left(\mathrm{Na}_{3} \mathrm{PO}_{4}\right)$ collected with see through reflective cavity adaptor as measured through a two-ply bag of white and brown kraft paper is shown in Figure 3. Although the spectrum in 3 (a) is dominated by fluorescence and Raman features from the paper layers, the library matching algorithm used is capable of reliably extracting the spectrum of trisodium phosphate from the packing layers and positively identifying it. 


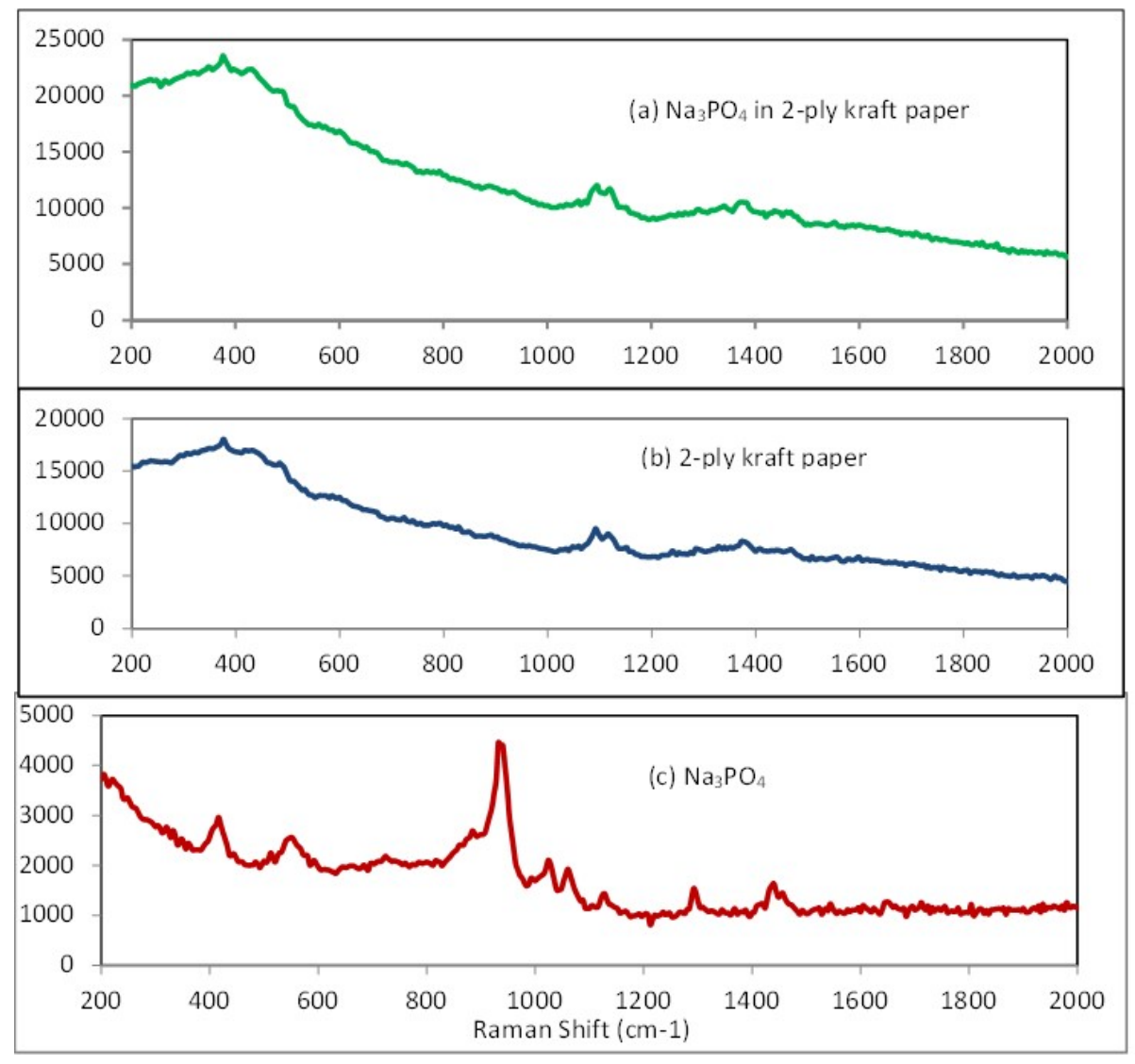

Figure 3: $1064 \mathrm{~nm}$ STRam identification of trisodium phosphate $\left(\mathrm{Na}_{3} \mathrm{PO}_{4}\right)$ in bilayer bag of white and brown kraft paper. (a) Spectrum measured through the bilayer bag using the STRaman technology; (b) spectrum of the bilayer bag measured with standard Raman configuration; (c) pure spectrum of $\mathrm{Na}_{3} \mathrm{PO}_{4}$.

\subsection{Large sampling area for more representative, repeatable results of solid samples}

Representative sampling of heterogeneous material to obtain bulk properties can be difficult by Raman spectroscopy, which typically has a sampling area of no more than $100 \mu \mathrm{m}$ in diameter. In multicomponent solid samples, uniform mixing of the ingredients on the scale of $100 \mu \mathrm{m}$ is difficult to achieve, especially when the grain size is this magnitude or greater. When solids are not well mixed on a scale smaller than the Raman sampling area, collection of spectra at different points on the sample can produce widely varying results due to the sample heterogeneity. This requires many measurements to be made and averaged for representative results for the bulk. A larger sampling area is needed to get more consistent measurements of typical products with multiple components. The difference in results from conventional Raman systems with a spot size of about $100 \mu \mathrm{m}$ vs. the larger sampling area of several $\mathrm{mm}$ with the STRaman probe can be seen in the spectra of a tablet of the over-the-counter pharmaceutical analgesic Excedrin ${ }^{\circledR}$ Migraine containing three active ingredients. Forty three spectra were collected over randomly chosen positions using a confocal fiber probe, which has a sampling spot diameter of roughly $0.3 \mathrm{~mm}$, with fifteen representative spectra overlaid in figure 4 (a). The Raman spectra of the three active ingredients - acetaminophen, aspirin and caffeine - can be detected in all the spectra, though their relative intensities vary greatly, reflecting the uneven distribution of the actives on this size scale. This heterogeneity makes it hard to reliably identify the product. This can be demonstrated by taking a number of such spectra, computing their average and using it to represent the product in a spectral library.. As shown in figure 4(b), using the conventional Raman with the $100 \mu \mathrm{m}$ sampling sport size there is a large variation in the hit quality index (HQI) against the reference spectrum in the library, reflecting the sample heterogeneity at this small scale. (The HQI is a measure of similarity based on the cross-correlation of a measured spectrum to a library spectrum, with a higher value indicating greater similarity). Using a passing threshold of 95,18 of the sample spectra result in false negatives. 
The average and standard deviation of the $43 \mathrm{HQI}$ values are 93.4 and 6.1, respectively. Using the STRaman approach with a spot size of about $4 \mathrm{~mm}$, each measurement is more representative of the bulk tablet properties. With the average and standard deviation of the HQI values at 99.89 and 0.07 , respectively, the reproducibility of the spectrum collected with the larger area, and therefore the identification is greatly improved, with no false negative results in the 17 measurements (Figures 4(c) \& (d)).
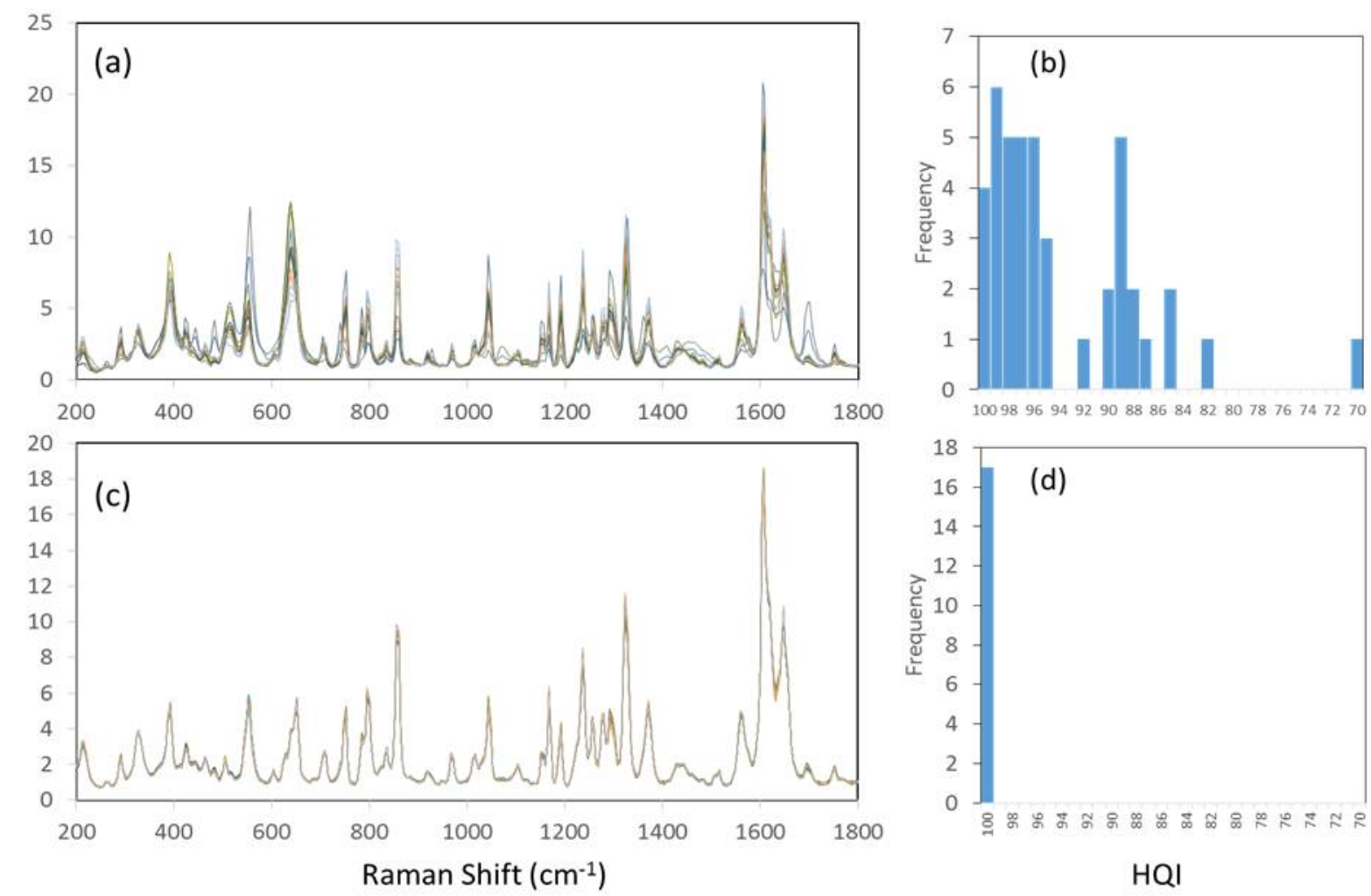

Figure 4: Comparative spectra of Excedrin migraine tablet as measured at various sample positions. (a) Overlay of 15 spectra acquired with conventional Raman configuration; (b) histogram of 43 HQI values with conventional Raman configuration. (c) Overlay of 15 spectra acquired with STRaman configuration; (d) histogram of 17 HQI values with STRaman configuration.

\subsection{Lower power density enables measurements of dark and sensitive samples}

Dark material absorbs light and can be a challenge to analyze with Raman without damaging the sample. With the design of the STRaman, laser power is distributed over a larger sampling area, as the laser power is defocused over a larger spot and thereby avoiding sample damage. With this system design, Raman technology is applicable to screening of sensitive samples such as photolabile or thermolabile material, biological tissues, historical art works or archaeological materials. Energetic materials such as gun powder must be very carefully handled and measured in very small quantities with conventional Raman because of the potential of ignition with the focused energy of the laser excitation. The defocused, large area design allows for collection of black gun powder spectrum at high laser power, as demonstrated in figure 5. Raman peaks of the gun powder component sulfur at $217.6 \mathrm{~cm}^{-1}$ and $471.5 \mathrm{~cm}^{-1}$, and nitrate at $1049.3 \mathrm{~cm}^{-1}$ as marked are clearly distinguished. 


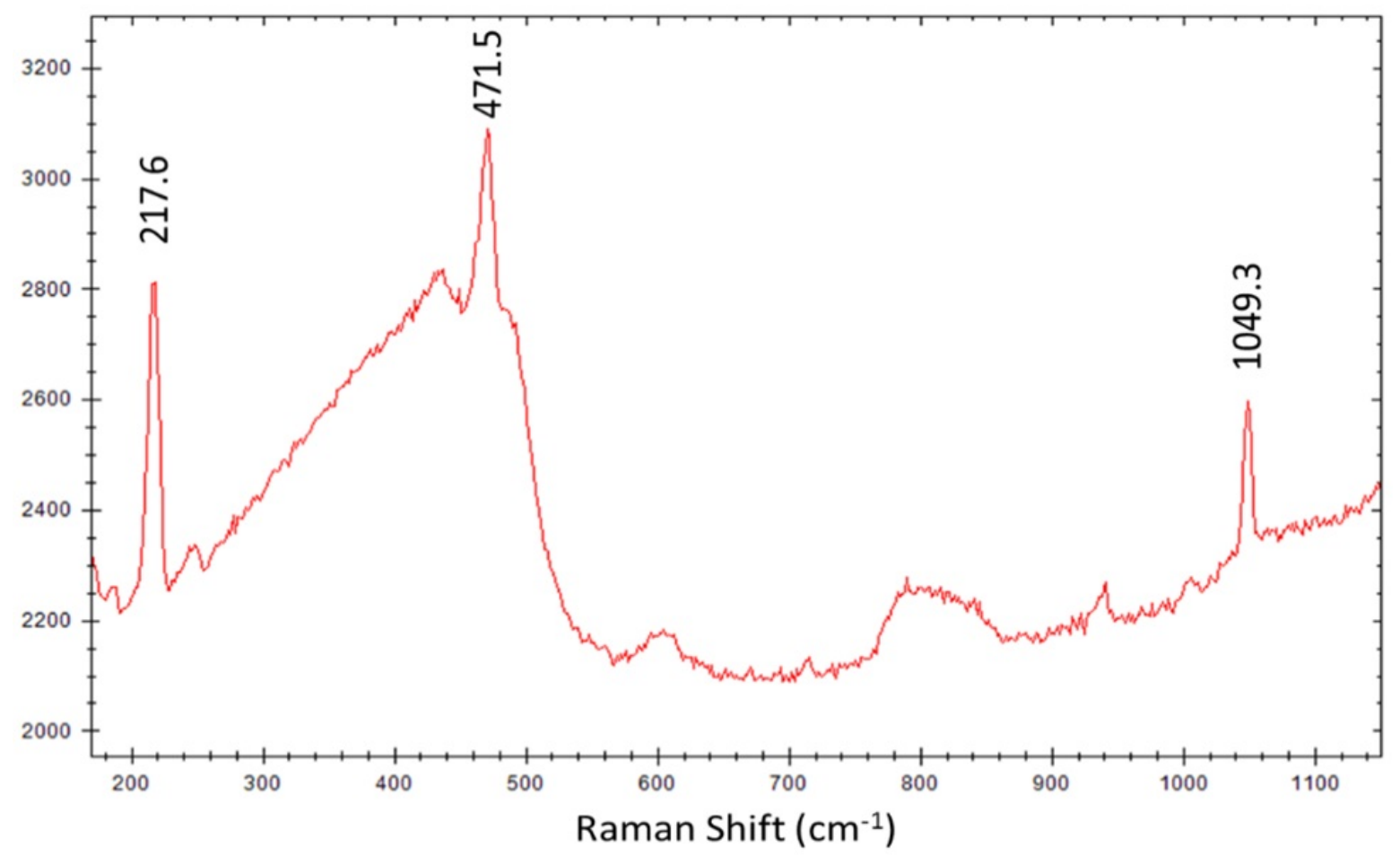

Figure 5: Raman spectrum of gun powder measured with 785-nm STRaman technology.

\subsection{Versatility of Sampling}

An additional advantage of this system design is the versatility of the system. The reflective cavity sampling adaptor can be configured for surface measurement when microanalysis on a sample surface is required. In this way the system is not limited to the large sample area, defocused power and beyond-surface measurements. It can be adapted to a confocal configuration. It can also be configured for confocal Raman with other adaptors allowing standoff, contact or microscopy analysis.

\section{CONCLUSIONS}

The ability to measure samples inside packages, eliminating the need for sample preparation, is one of the major advantages of Raman. The advancements brought forth with STRaman technology take that a step further to measuring through opaque packages - from white plastic bottles to fiber sacks, to kraft paper sacks, envelopes and even skin allows easy adoption of this spectroscopic tool in many working environments, in the laboratory or in the field. The large sampling area and decreased power density of the laser increase the ability of Raman for measurement of sensitive samples, and provides more reliable sampling of heterogeneous solids. The application of the technology for both 785 $\mathrm{nm}$ and $1064 \mathrm{~nm}$ laser excitation addresses a diversity of samples, including dark and highly colored packaging that are influenced by fluorescence. This expands Raman to many new potential users, for whom it has not previously been a viable tool.

\section{REFERENCES}

[1] Mogilevsky, G., Borland, L., Brickhouse, M. and Fountain, A.W., " Raman Spectroscopy for Homeland Security Applications,” Intl. J. Spectroscopy, Volume 2012, Article ID 808079, 12 pages (2012). http://dx.doi.org/10.1155/2012/808079. 
[2] Hargreaves, M.D., "Handheld Raman Spectrometers and Their Applications", In: R.A. Meyers, editor. [Encyclopedia of Analytical Chemistry: Applications, Theory and Instrumentation], John Wiley and Sons, Chichester, UK, 1-16 (2014).

[3] Zhao, J. Zhou, X.J. and Wang, S.X., "Methods and devices for measuring Raman scattering of a sample". US patent 10,113,969 B2 (2018).

[4] Zhao, J., Bakeev, K.A., Zhou, J. "Raman Spectroscopy Peers Through Packaging". Photonic Spectra. Feb. 2018, https://www.photonics.com/Articles/Raman_Spectroscopy_Peers_Through_Packaging/a62932

[5] Matousek, P. et al. "Subsurface probing in diffusely scattering media using spatially offset Raman spectroscopy," Appl Spectrosc, 59, 393-400 (2005). 